\title{
SIMULATION OF CLUSTER IMPACT INDUCED DESORPTION AND COOLING
}

\section{R.P.Webb*}

Surrey Ion Beam Centre

Advanced Technology Institute

University of Surrey

GU2 7XH, UK

\section{ABSTRACT}

Energetic impact of $\mathrm{C}_{60}$ fullerene molecules on surfaces are beginning to find a number of applications. In particular the scattering of fullerene molecules from surfaces has for some time now been a good method of determining the mechanical properties of the molecular cages. But also the use of $\mathrm{C}_{60}$ and other molecular ions in secondary mass spectrometry (SIMS) as a probing beam is showing great promise for the analysis of large organic molecules.

Molecular dynamics computer simulation is used here to investigate the behaviour of a surface after an impact with a $\mathrm{C}_{60}$ molecule. In particular the simulations will concentrate on how the disruption caused to the surface can cause the ejection of a loosely bound adsorbate molecule close to the impact site. In general simulations of these kind of experiments have led to a better understanding of the processes involved and how a surface responds in very much a different way after a cluster impact than that due to a single atom impact. When a cluster impacts a surface there is a better mechanism to create co-ordinated motion across the surface. In some cases a travelling acoustic wave can be created. The co-ordinated motion can gently push a large molecule from a surface. In the case of single atom impacts the motion caused in the surface region is often more discrete and results in the removal of a large molecule in fragments. It is this difference in behaviour that has made the cluster SIMS so successful for the analysis of large organic molecules.

The simulated desorption process of hot adsorbates results in a 10\% cooling of the molecules on ejection. Unlike the sputtering process this desorption process does not require that the binding energy of the ejected material is all removed from the particle after ejection.

\section{INTRODUCTION}

Molecular Dynamics simulations of the sputtering process have been used successfully for more than 40 years $^{1}$ to help understand the complex physical phenomena that occur after the impact of an energetic particle on a solid surface. There has been a long history using and improving the simulation models to gain greater insight over that time. Since 1992 we have been modelling the interaction of $\mathrm{C}_{60}$ molecules with surfaces $^{2,3}$. By careful comparisons with experiments, performed in collaboration with the University of Karlsruhe, the simulation models have been verified for both low energy scattering of the molecules ${ }^{4}$ and the surface damage created from the impact ${ }^{5}$. The model has been used to investigate how the deformation of the surface caused by the 
impact of a large molecule such as $\mathrm{C}_{60}$ can cause the ejection of loosely bound molecules from the surface ${ }^{6,7}$. Most recently it has been shown computationally how the deformation of a silicon surface caused by a high energy $\mathrm{C}_{60}$ impact can eject a large polystyrene molecule without fragmentation of the polystyrene molecule ${ }^{8}$. This is of particular interest to the Secondary Ion Mass Spectrometry community who are starting to use molecular - largely $\mathrm{C}_{60}, \mathrm{SF}_{5}$ and $\mathrm{Au}_{3}$ - and cluster beams as sputtering beams because of their ability to produce high sputtering yields without causing damage deep in the target. They are also very attractive to use as they produce a much higher fraction of intact large molecular species instead of the normal fragments when sputtering organic films. Understanding the intact molecular ejection mechanisms is fundamental to optimising the yield of such molecules. Simulations have shown that the original concept that the impacting $\mathrm{C}_{60}$ shatters on impact and scatters across the surface causing substantial molecular ejection is incorrect. The simulations make it quite clear that the fullerene impact causes co-ordinated motion in the target material which propagates out from the impact site and lifts any surface molecules, pushing them gently away from the surface, this can occur with sufficient energy transfer to gently eject the molecule intact from the surface. This simulation study is still very much in the early stages. A more detailed analysis of the behaviour of the co-ordinated motion with respect to the impact parameters of energy, angle, cluster size, target material and structure could provide useful input into an experimental programme. The work reported here forms the first part of a more detailed study of the behaviour of adsorbed molecules close to the impact point of an energetic cluster. The simulations presented here do not demonstrate the behaviour of a typical system that might be used in a SIMS system. The target material used here is graphite, which has very anisotropic elastic properties, in particular the surface layer acts as a vibrating membrane when struck. This is unlikely to be the case in other more isotropic materials such as silver or silicon. Also the adsorbate molecule, fullerene, is atypically robust. However the results presented demonstrate interesting potential deorption processes that could be exploited. The effect of the robustness of the adsorbate molecule on these processes will be the subject of a further study.

The simulation model employed in this study has been described in detail in the past ${ }^{9}$. In brief we employ a Molecular Dynamics scheme to calculate the many body interactions of 150,000 carbon atoms with each other. We use the Brenner many-body hydro-carbon potential ${ }^{10}$ to describe the intermediate distance inter-atomic interactions, this is splined to the $\mathrm{ZBL}^{11}$ Coloumb style potential at close separations. We use an adapted Brenner 
potential ${ }^{12}$ to describe the long range interactions so that the graphite modelled exhibits inter-layer bonding and allows molecules to become bound in a Van der Waals fashion to the modelled graphite surface. The computation employs periodic boundary condition in the lateral dimensions ( $x$ and $z$ ) and free boundaries in the direction normal to the surface (y). Figure 1 shows the potential energy of a $\mathrm{C}_{60}$ fullerene molecule as a function of height above the modelled graphite surface using this scheme. The potential goes through a minimum at a height of about $0.62 \mathrm{~nm}$ from the centre of the molecule and gives an effective binding energy of $0.275 \mathrm{eV}$. The atoms of both the graphite and fullerene molecule are positioned at their equilibrium positions and then the fullerene is brought closer to the surface and the potential energy of the fullerene is calculated. The difference between the Potential Energy at infinity ( $\sim 0.9 \mathrm{~nm}$ in this case!) and the minimum gives the binding energy of the molecule and is shown on figure 1 .

Minimization is also performed in $\mathrm{x}$ and $\mathrm{z}$ directions, although this is much less pronounced. A single fullerene molecule is placed at the minimum position in the central portion of the computed crystal. A second fullerene is placed at $1 \mathrm{~nm}$ above the surface and given kinetic energy appropriate for a range of different energy impacts. The simulation is followed for $4 p s$ which is long enough for the surface acoustic wave to pass underneath the first fullerene molecule and not too long such that reflections from the boundaries of the simulation should amplify and distort the results of the simulations. To investigate the efficiency of this acoustic wave in desorbing molecules from a surface a number of different impacts have been simulated at various distances from the centre of the adsorbed fullerene for a range of different impact energies. A set of 25 different impacts have been simulated at 5 different radial distances from the adsorbed molecule. The perpendicular velocity of the initially adsorbed fullerene is recorded and from this it is relatively easy to determine if the molecule is desorbed.

\section{RESULTS \& DISCUSSION}

Figure 2 shows the impact points chosen around the position of the initially adsorbed fullerene molecule. The large central circle represents the position of the adsorbed molecule and the surrounding circles the impact sites of the $5 \mathrm{keV}$ fullerene. As can be seen the 25 trajectories fall into 5 circular bands around the adsorbed molecule.

The figure also shows the impact points from which the initially adsorbed fullerene becomes desorbed. One might expect that the closer to the adsorbed molecule the more likely it is to desorb it. The desorption takes place when the impacting fullerene causes an 
acoustic wave to spread out from the impact site imparting enough energy to the adsorbed molecule for it to leave the surface ${ }^{13}$. It is reasonable to expect that this wave will lose energy as it propagates across the surface. Hence the further the wave must travel across the surface the less likely it is to cause ejection. This is borne out to some degree from these results in that there is a higher probability of ejection from impacts in the $2^{\text {nd }}$ and $3^{\text {rd }}$ rings than those in the $4^{\text {th }}$ and $5^{\text {th }}$ rings. The more interesting effect is seen with impacts in the $1^{\text {st }}$ ring. At energies above $1 \mathrm{keV}$ none of these collisions result in desorption of the fullerene. It is reasonable to expect that if the impact is too close to the fullerene it will hit it and potentially break it, thus preventing it from escaping the surface. This does not happen here, the position of the impact was chosen specifically to prevent this from happening. The impact is far enough away that it should not directly impart energy to the initially adsorbed fullerene to desorb it. Instead what happens is that the surface around the impact site of the impacting fullerene is broken by the impact and consequently the atoms around the crater are now under co-ordinated. The consequence of this is that these atoms can become much more attractive to the adsorbed fullerene molecule and as the surface vibration interacts with the adsorbed fullerene it now brings these under co-ordinated crater atoms close. They effectively pull the adsorbed fullerene down into the crater and more firmly attach it to the surface preventing it from leaving. In these simulations impacts at $1 \mathrm{keV}$ are very efficient at desorbing the loosely bound molecules. The acoustic wave created by the impact at this energy has more energy than at lower energies and can hence travel further and have a better chance of knocking off a loosely bound molecule. Impacts with energies higher than this will tend to penetrate deeper into the surface of the graphite, create more surface damage and so not produce such a strong surface acoustic wave. It should be noted that this behaviour is very much a property of the layered structure of graphite. In a more three dimensional material such as silver or silicon this mechanism will not be so prevalent.

In figure 3 typical perpendicular velocities of the loosely bound molecules as a function of time from $1 \mathrm{kev}$ initial impacts at different ring radii are shown. The positive direction is into the target and negative direction is away from the surface. It can be seen that the adsorbed molecule is initially pushed away from the surface as the acoustic wave passes. This is shown by a small minimum in each of the curves. The time at which this occurs is later the greater the distance away of the impact. Once the initial wave passes the adsorbate the surface is lowered and the adsorbate is pulled down towards the surface as is seen by the perpendicular velocity becoming positive. A short time later the surface 
recovers and rises and collides with the adsorbate whilst it is still being pulled to the surface. The result is that the molecule reverses its direction - the velocity now becomes negative - with sufficient velocity that it can now overcome its initial binding to the surface - the minimum - and the particle eventually flies free of the surface - the constant velocity portion at longer times. All of this is much easier to observe in movies that have been made of these sequences.

In figure 4 the same time development of the perpendicular velocities are shown for different energy impacts in the second ring. This figure shows that the $1 \mathrm{keV}$ and $2 \mathrm{keV}$ impacts behave in a similar fashion, but at higher energies, $5 \mathrm{keV}, 10 \mathrm{keV}$ and $20 \mathrm{keV}$ the loosely bound fullerene molecule is desorbed promptly by the initial passage of the acoustic wave as it passes underneath the molecule.

In figure 5 the time development of the perpendicular velocity of the adsorbate is shown for impacts of $5 \mathrm{keV}$ for different impact rings. This is comparable to figure 3 for the $1 \mathrm{keV}$ impacts. The difference here is the clear split in the mechanism of the desorption process. For impacts in the $4^{\text {th }}$ and $5^{\text {th }}$ rings the desorption process is similar to that of the $1 \mathrm{keV}$ impacts. For impacts in the $2^{\text {nd }}$ and $3^{\text {rd }}$ rings the desorption process occurs as soon as the acoustic wave passes underneath it. Clearly the wave looses "desorbing power" as it travels across the surface. So that nearby it has enough power to desorb a molecule as it passes, but further away the molecule is desorbed by the surface depression after the crest of the wave has passed. Impacts in the inner most ring at this energy cause substantial break-up of the surface and, as described above, cause the loosely bound fullerene molecule to become more tightly bound to the surface as can be seen by the substantial velocity changes that the molecule experiences, but yet still remains firmly attached to the surface.

A number of simulations were accidentally run with the atoms of the adsorbed fullerene not at their equilibrium position. The result of this is that they had an initial vibrational breathing mode. The amplitude of this mode can be seen in figure 6 to be about $\pm 0.05 \AA$. Figure 6 shows the radius of the adsorbed fullerene as a function of time after a $5 \mathrm{keV}$ impact. The molecule shows a strong breathing mode characteristic of a temperature of about $1200 \mathrm{~K}$. Approximately $100 \mathrm{fs}$ after the impact of the $5 \mathrm{keV}$ fullerene the amplitude of the breathing mode changes. This is at the same time that the molecule is desorbed from the surface by the acoustic wave passing underneath it. The unexpected thing is that the molecule after being desorbed by what is really a collisional mechanism has a reduced internal energy and the amplitude of the breathing mode is reduced by $\sim 10 \%$ to 
approximately $1100 \mathrm{~K}$. Figure 7 shows the same behaviour for the slower two step desorption process and interestingly shows a similar decrease in amplitude after desorption. The likely explanation for this is that as the surface strikes the loosely bound fullerene it dampens, slightly, the breathing mode and might even transfer some of this energy into the desorption process itself. Contact with the "cold" surface has reduced the thermal vibrational amplitude of the adsorbate molecule as it desorbs. The disruption and general vibrational modes introduced by the colliding fullerene close by make it impossible to track precisely where the energy from this cooling process has gone to in the target surface.

In conclusion the desorption behaviour of adsorbed molecules on surfaces arising due to energetic cluster or molecule impact is not necessarily as obvious as it might first seem. The ejection mechanism from the induced surface acoustic wave could possibly have been predicted relatively easily, but the influence of the hole punched by the impacting molecule in preventing the desorption process from occurring adds a new complexity to this behaviour and the reduction in the breathing mode amplitude after desorption seems to be quite surprising at first sight.

\section{References}

1) For example see the reviews: D.E. HARRISON Jr., CRC Critical Reviews in Solid State \& Materials Science, 14(1), (1988), S1. and D.E. HARRISON Jr. Radiation Effects, 70, (1983), 1.

2) R. SMITH \& R.P. WEBB, Proc. R. Soc. Lond. A 441, (1993), 495.

3) R.P. WEBB, R. SMITH, E. DAWNKASKI, B. GARRISON \& N. WINOGRAD, Int. Vid. J. of Eng. Res. 3, (1993), 63.

4) M. HILLENKAMP, J. PFISTER, M. KAPPES \& R.P. WEBB, J. Chem. Phys. 111(22), (1999), 10303.

5) R.P. WEBB, M. KERFORD, M. KAPPES \& G. BRAUCHLE, Nucl. Instrum. \& Meths. B122, (1997), 318.

6) M. KERFORD \& R.P. WEBB, Nucl. Instrum. \& Meths. B180, (2001), 44.

7) R.P. WEBB, M. KERFORD, E. ALI, M. Dunn, L. KNOWLES, K. LEE, J. MISTRY \& F. WHITEFOOT, Surf. Interface Anal. 31, (2001), 297.

8) Z. POSTAWA, Proc. SIMS XIV, San Diego, (2003) in press.

9) R. SMITH, D.E. HARRISON Jr., B.J. GARRISON, Phys. Rev. B40, (1989), 93.

10) D.W. BRENNER, Phys. Rev. B42, (1990), 9458. and D.W. BRENNER, Phys. Rev. B46, (1992), 1948. 
11) J. ZIEGLER, J. BIERSACK \& U. LITTMARK, The Stopping and Ranges of Ions (Pergamon,

New York, 1985)

12) R. SMITH \& K. BEARDMORE, Thin Solid Films, 272, (1996), 255.

13) R.P.WEBB, Applied Surf. Sci. 231-232, (2004), 59.

\section{Figure Captions}

Figure 1: Potential Energy of adsorbed $\mathrm{C}_{60}$ on graphitic surface as a function of height

Figure 2: Summary of the 25 different impact sites for each of the different energy impacts of fullerene that are used in the simulations. The tables are split into trajectory sets which are from rings shown in the diagrams. The rings get progressively further away from the initial position of the adsorbate. "Y" means that the adsorbate is desorbed after the impact. "N" means it remains attached to the surface.

Figure 3: Perpendicular velocity of a loosely bound fullerene molecule as a function of time after impact by a $1 \mathrm{keV}$ fullerene molecule at different distances. The positive direction is into the surface, towards the bulk, and the negative direction is away from the surface and into the vacuum

Figure 4: Perpendicular velocity of a loosely bound fullerene molecule as a function of time after impact by a fullerene molecule of different energies from ring 2 . The positive direction is into the surface, towards the bulk, and the negative direction is away from the surface and into the vacuum

Figure 5: Perpendicular velocity of a loosely bound fullerene molecule as a function of time after impact by a $5 \mathrm{keV}$ fullerene molecule at different distances. The positive direction is into the surface, towards the bulk, and the negative direction is away from the surface and into the vacuum

Figure 6: Radius of the loosely bound fullerene molecule as a function of time after impact by a $5 \mathrm{keV}$ fullerene molecule in $2^{\text {nd }}$ ring. Initially the loosely bound molecule is "hot" and exhibits a breathing mode. After the surface collides with it and ejects it ( 100fs), the breathing mode amplitude is reduced. There is no change in frequency.

Figure 7: Radius of the loosely bound fullerene molecule as a function of time after impact by a $5 \mathrm{keV}$ fullerene molecule in $4^{\text {th }}$ ring. Initially the loosely bound molecule is "hot" and exhibits a breathing mode. After the surface collides with it and ejects it $(\sim 1100 f s)$, the breathing mode amplitude is reduced. There is no change in frequency. 


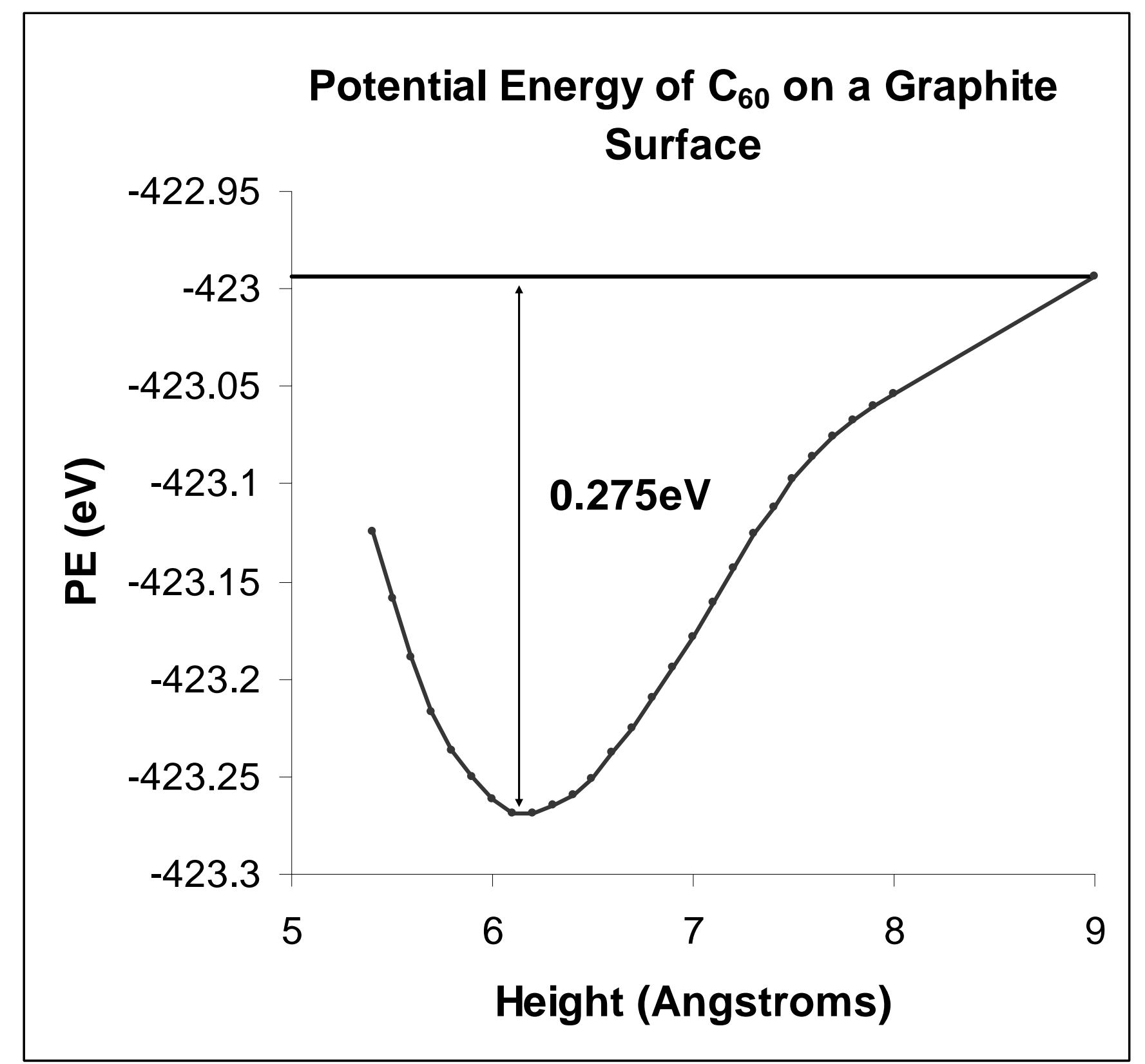

Figure 1 


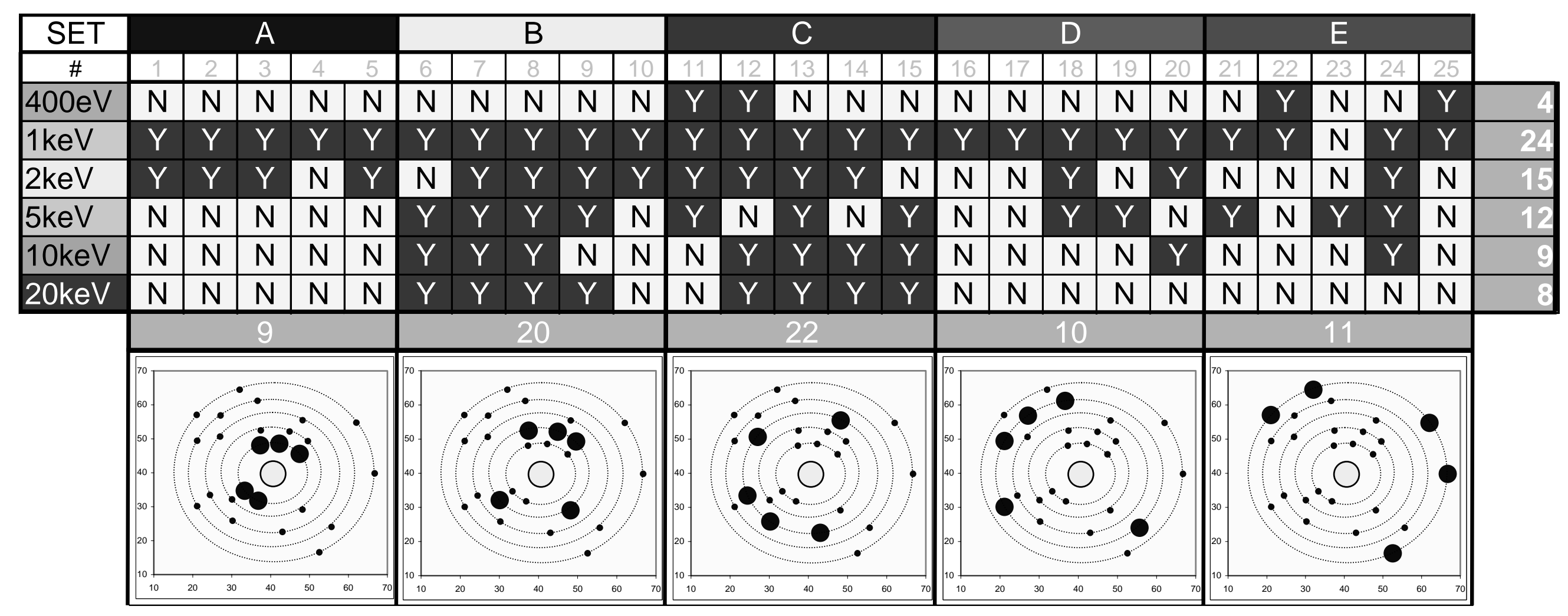

Figure 2 


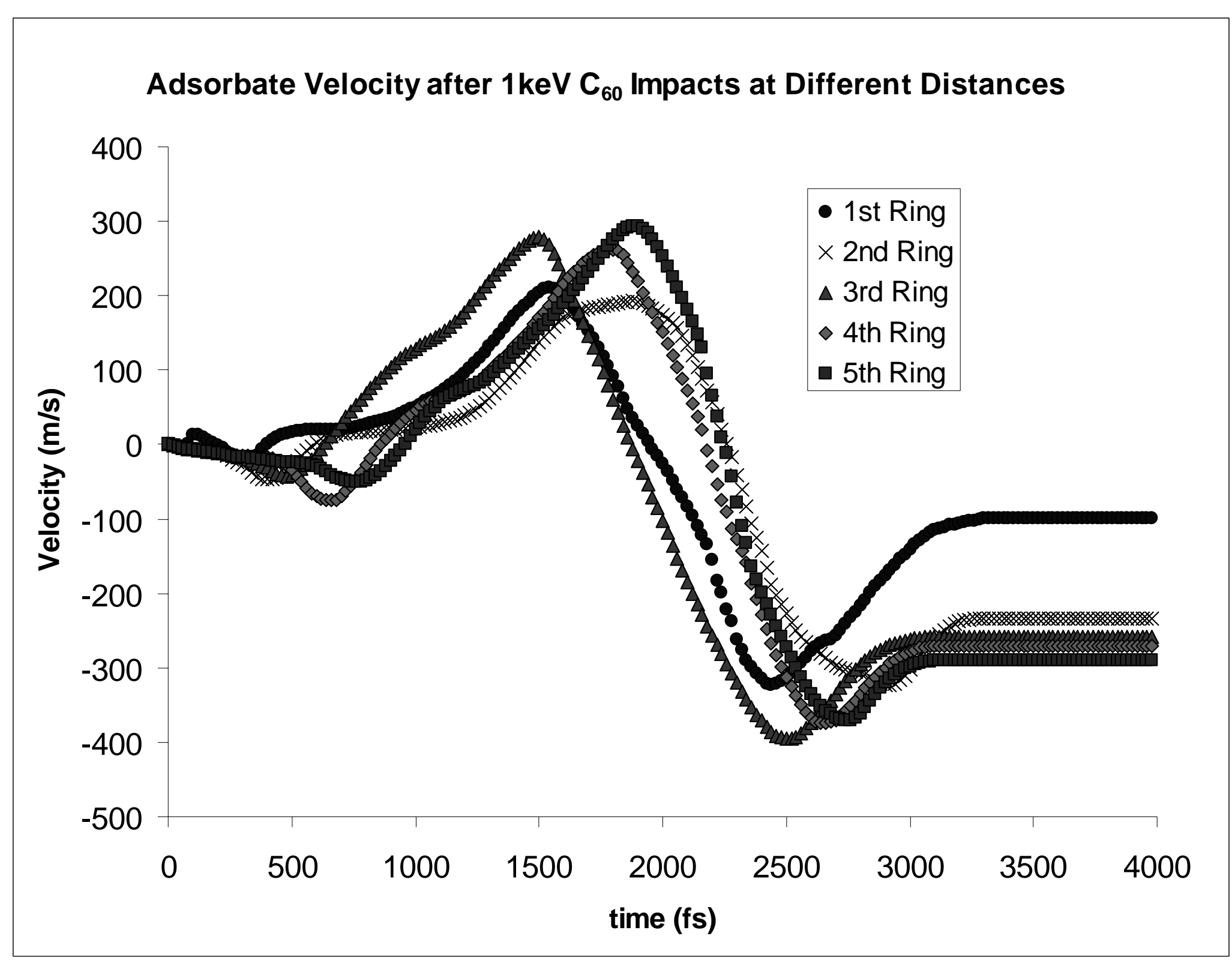

Figure 3 


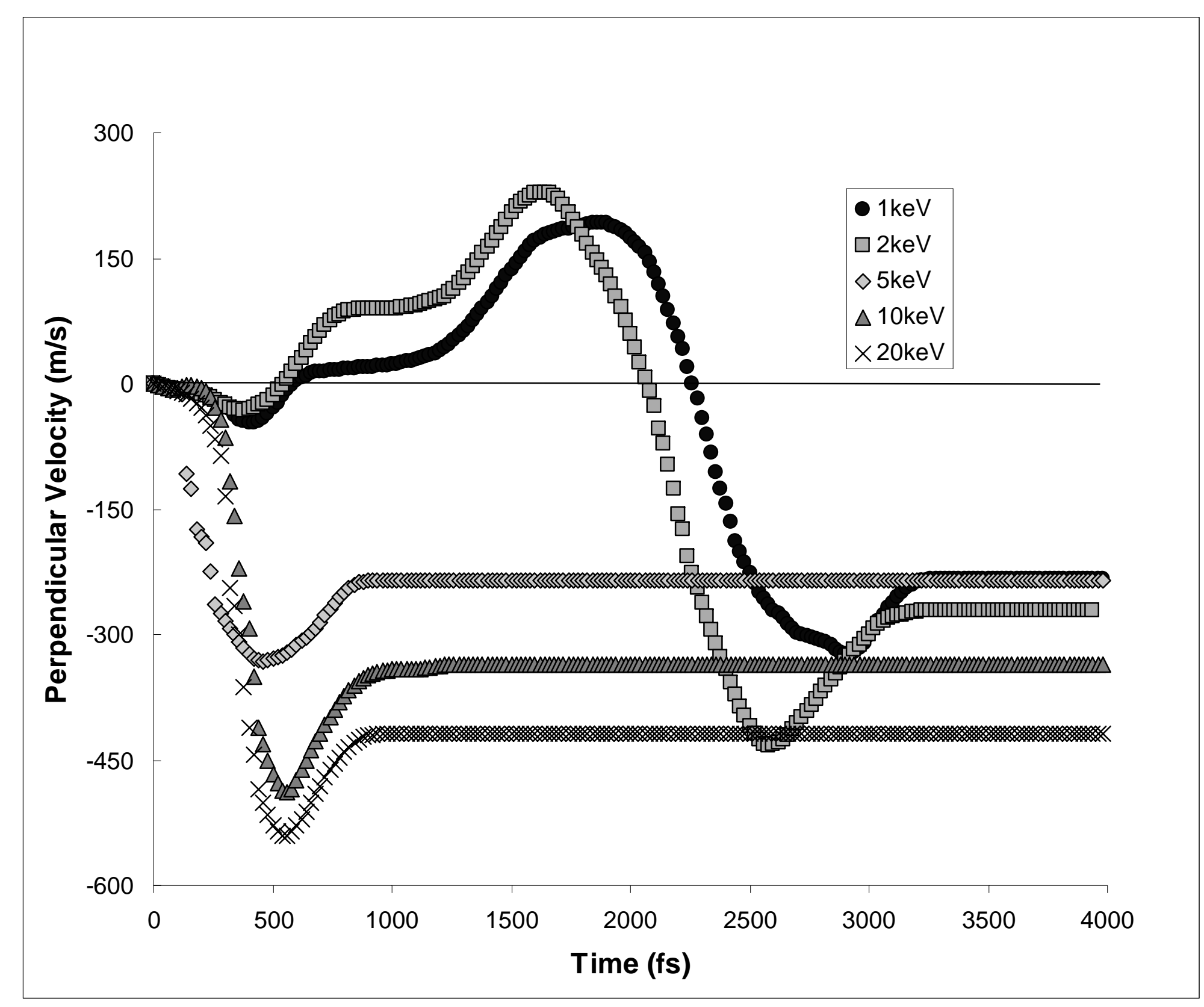

Figure 4 


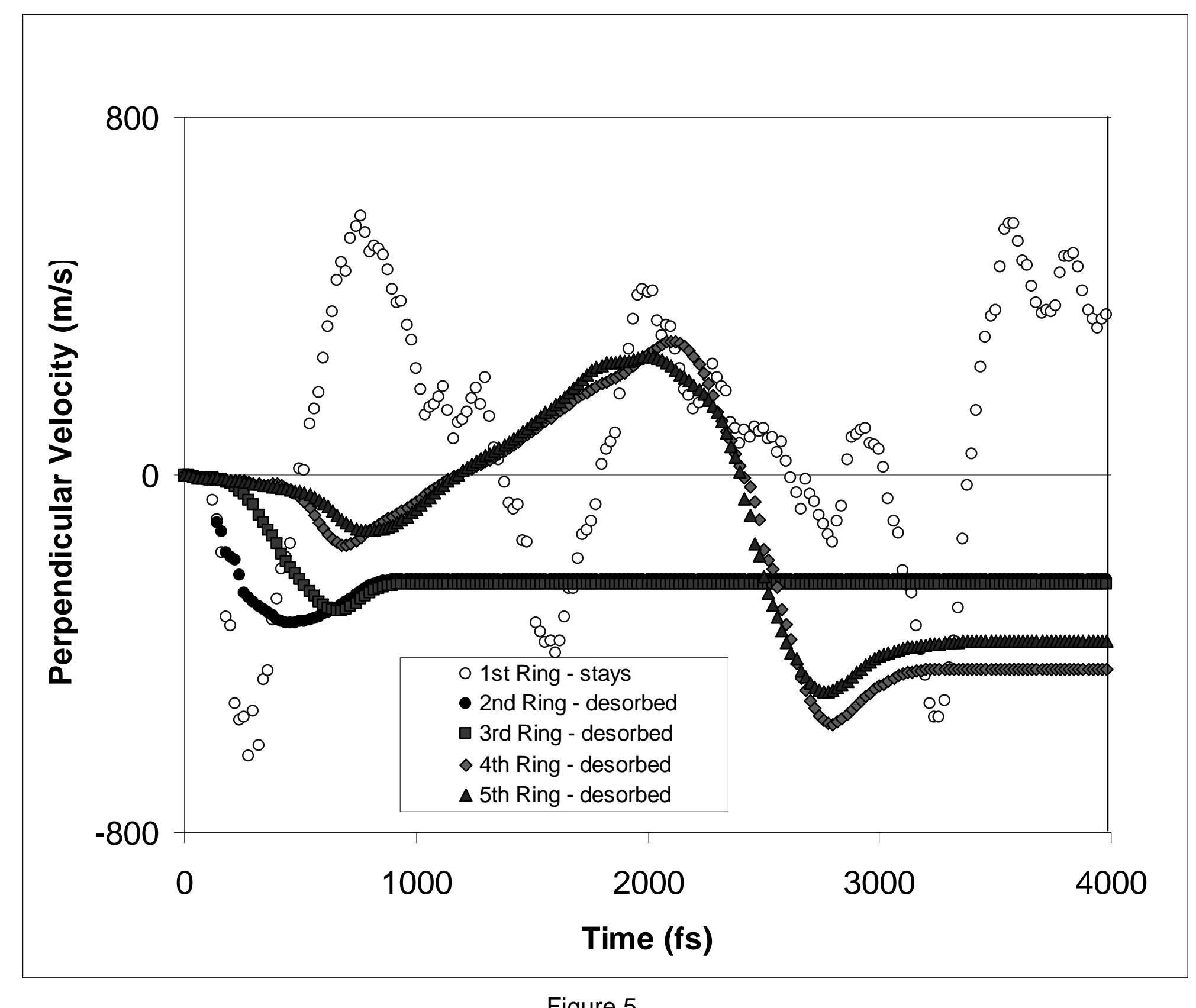




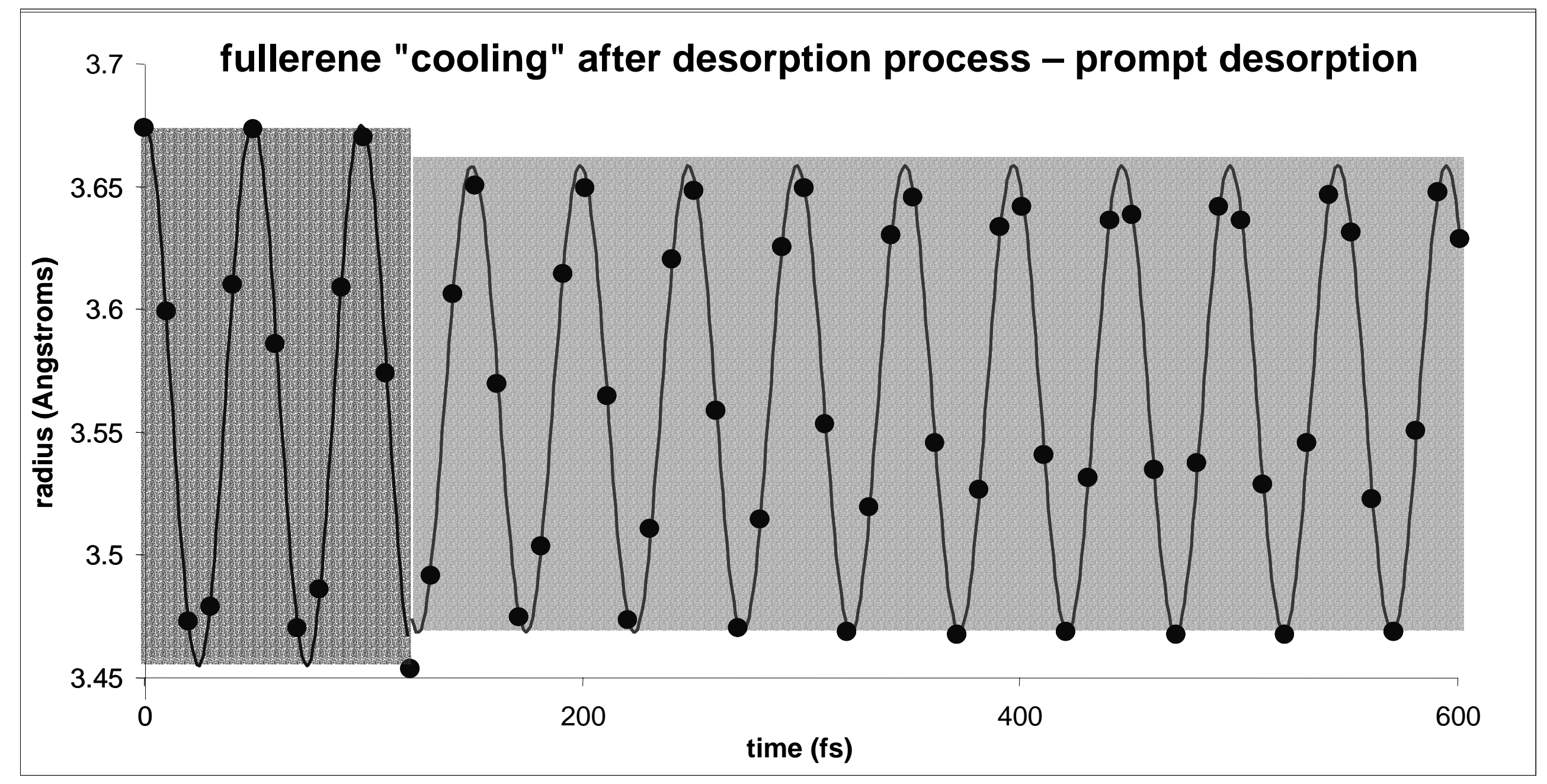

Figure 6 


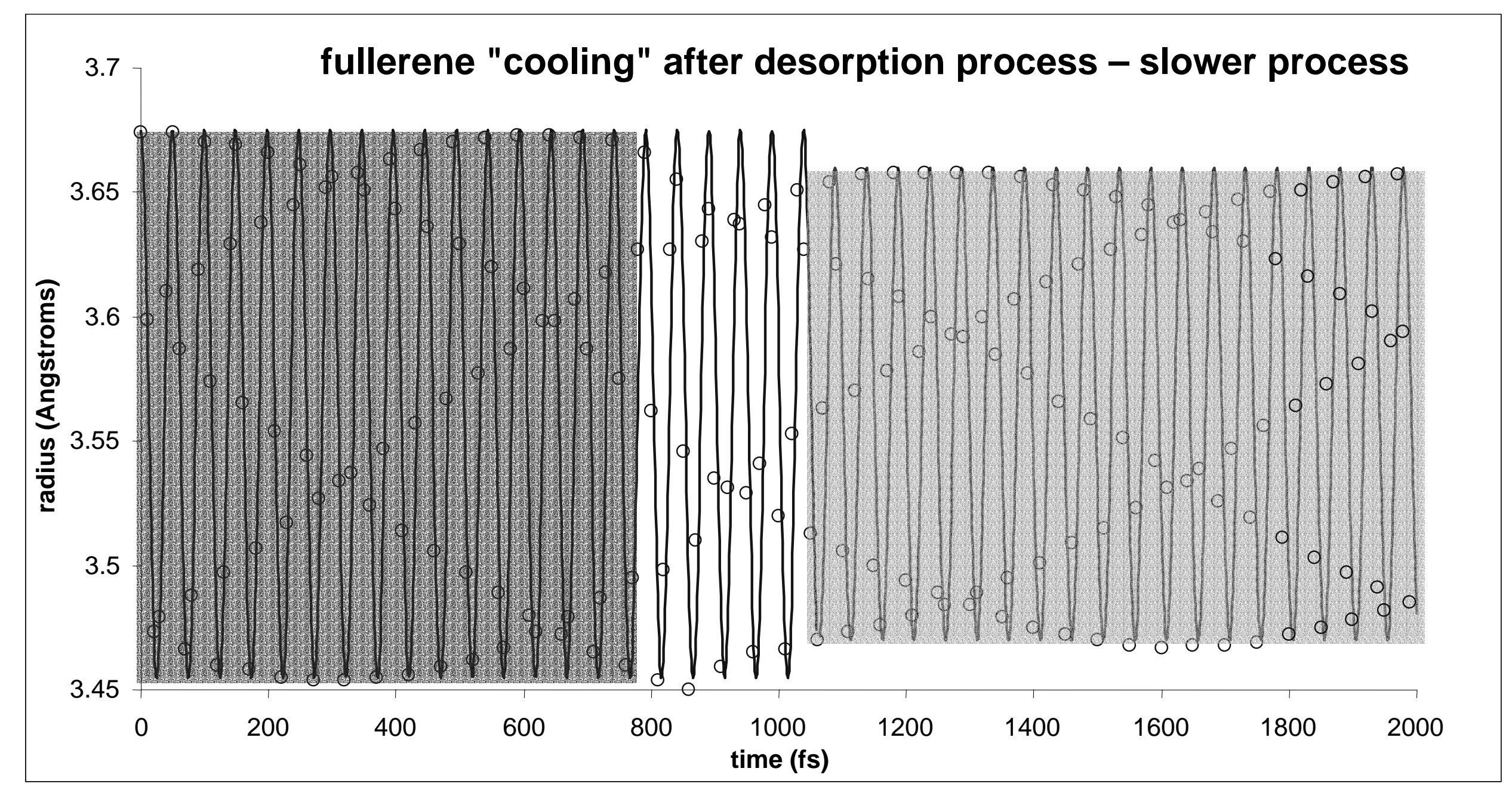

Figure 7 